Ferrata Storti Foundation

\title{
Glycoprotein Ib clustering in platelets can be inhibited by $\alpha$-linolenic acid as revealed by cryo-electron tomography
}

Simona Stivala, ${ }^{1 *}$ Simona Sorrentino ${ }^{2 *}$ Sara Gobbato, ${ }^{1}$ Nicole R. Bonetti ${ }^{1,3}$ Giovanni G. Camici, ${ }^{1,4,5}$ Thomas F. Lüscher, ${ }^{1}$ Ohad Medalia ${ }^{2,6}$ and Jürg H. Beer ${ }^{1,3}$

${ }^{1}$ Laboratory for Platelet Research, Center for Molecular Cardiology, University of Zurich,

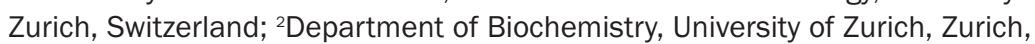
Switzerland; IInternal Medicine, Cantonal Hospital of Baden, Baden, Switzerland; ${ }^{4}$ University Heart Center, University Hospital Zurich, Zurich, Switzerland; ${ }^{5}$ Department of Research and Education, University Hospital Zurich, Zurich, Switzerland and ${ }^{6}$ Department of Life Sciences and the National Institute for Biotechnology in the Negev, Ben-Gurion University, Beer-Sheva, Israel

*SSt and SSo contributed equally as co-first authors

\section{ABSTRACT}

D latelet adhesion to the sub-endothelial matrix and damaged endothelium occurs through a multi-step process mediated in the initial phase by glycoprotein Ib binding to von Willebrand factor (vWF), which leads to the subsequent formation of a platelet plug. The plant-derived $\omega-3$ fatty acid $\alpha$-linolenic acid is an abundant alternative to fish-derived n-3 fatty acids and has anti-inflammatory and antithrombotic properties. In this study, we investigated the impact of $\alpha$-linolenic acid on human platelet binding to vWF under high-shear flow conditions (mimicking blood flow in stenosed arteries). Pre-incubation of fresh human blood from healthy donors with $\alpha$ linolenic acid at dietary relevant concentrations reduced platelet binding and rolling on vWF-coated microchannels at a shear rate of $100 \mathrm{dyn} / \mathrm{cm}^{2}$. Depletion of membrane cholesterol by incubation of platelet-rich plasma with methyl- $\beta$ cyclodextrin abrogated platelet rolling on vWF. Analysis of glycoprotein Ib by applying cryo-electron tomography to intact platelets revealed local clusters of glycoprotein $\mathrm{Ib}$ complexes upon exposure to shear force: the formation of these complexes could be prevented by treatment with $\alpha$-linolenic acid. This study provides novel findings on the rapid local rearrangement of glycoprotein Ib complexes in response to high-shear flow and highlights the mechanism of in vitro inhibition of platelet binding to and rolling on vWF by $\alpha$-linolenic acid.

\section{doi:10.3324/haematol.2019.220988}

Check the online version for the most updated information on this article, online supplements, and information on authorship \& disclosures: www.haematologica.org/content/105/6/1660

(C)2020 Ferrata Storti Foundation

Material published in Haematologica is covered by copyright. All rights are reserved to the Ferrata Storti Foundation. Use of published material is allowed under the following terms and conditions:

https://creativecommons.org/licenses/by-nc/4.0/legalcode. Copies of published material are allowed for personal or internal use. Sharing published material for non-commercial purposes is subject to the following conditions: https://creativecommons.org/licenses/by-nc/4.0/legalcode, sect. 3. Reproducing and sharing published material for commercial purposes is not allowed without permission in writing from the publisher.

\section{Introduction}

The first event leading to the formation of a platelet plug is mediated by the glycoprotein Ib-IX complex (GpIb-IX), the second most-abundant platelet receptor after the integrin $\alpha \mathrm{IIb} \beta 3 .^{1.3}$ Platelet binding to von Willebrand factor (vWF) is tightly controlled in order to occur only at sites of bleeding but not in the normal circulation, where it would cause thrombosis. This regulation involves activation of vWF only at high flow rates and binding of GpIb to the A1 domain of vWF through a two-step mechanism, in which a vWF multimer first elongates, then the A1 domain transitions to a high-affinity state. ${ }^{4.6}$ The role of high-shear flow in the pathogenesis of thrombosis is particularly relevant under pathological conditions such as in stenosed, atherosclerotic arteries, where shear stress can increase above $100 \mathrm{dyn} / \mathrm{cm}^{2}$ (shear rate $>4000 / \mathrm{s}$ ). ${ }^{7.8}$ Because of its pivotal role in initiating platelet adhesion, $\mathrm{GpIb}$ represents a promising antithrombotic target.

Omega-3 fatty acids (n-3 FA) are a class of naturally occurring polyunsaturated fatty acids that include the plant-derived $\alpha$-linolenic acid (ALA), whose cardioprotective effects have been shown by us and others, ${ }^{9 \cdot 12}$ which is readily available and marine-derived n-3 FA, whose use is restricted by limited fishery resources and sea pollution. ${ }^{13-15} \mathrm{n}-3 \mathrm{FA}$ modulate cellular responses through incorporation into plasma 
membranes and reduction in the formation of typical protein complexes/lipid rafts, among other effects. ${ }^{16-18}$ Based on our previous observations that ALA reduces platelet reactivity, ${ }^{10,19}$ and on studies showing the presence of $\mathrm{GpIb}$ in lipid rafts, ${ }^{20-22}$ we hypothesized that ALA might interfere with the distribution of GpIb on the plasma membrane in high-shear flow and, therefore, alter binding to vWF.

\section{Methods}

\section{Blood samples}

Blood from healthy volunteers was obtained from the Blood Center of the Swiss Red Cross at the Cantonal Hospital Baden with informed consent according to the Declaration of Helsinki. The study was approved by the Institutional Review Board. EDTA or citrated blood was kept at room temperature until assays were performed (within $2 \mathrm{~h}$ of drawing). Blood was incubated with vehicle $(0.1 \%$ ethanol) or ALA $30 \mu \mathrm{M}$ for $1 \mathrm{~h}$ at room temperature before being used for subsequent experiments. The n-3 FA dose was chosen based on a previous study showing this to be a dietary reachable concentration. ${ }^{23}$ Platelet adhesion to vWF was performed on a Bioflux 200 system (Fluxion Bioscience, San Francisco, CA, USA) according to the manufacturer's protocol (see the Online Supplementary Data file for details).

\section{Immunofluorescence staining for ground state deple- tion microscopy}

Washed platelets isolated from vehicle or ALA-treated samples were fixed with $4 \%$ paraformaldehyde for $15 \mathrm{~min}$, then spun on a 1.5 coverslip in a Cytospin (Thermo Fisher Scientific, Waltham, MA, USA) and stained for ground state depletion (GSD) microscopy. For details, see the Online Supplementary Data file.

\section{Flow cytometric analysis of von Willebrand factor binding}

Platelet-rich plasma or washed platelets from vehicle- or ALAtreated blood samples were fixed with $4 \%$ paraformaldehyde for $15 \mathrm{~min}$, then incubated with $1 \%$ bovine serum albumin (BSA) in phosphate-buffered saline (PBS) containing a rabbit anti-human vWF antibody (1:500, Dako A0082) and an antiGpIbo-APC (BD Bioscience, San Jose, CA, USA) for $1 \mathrm{~h}$. In some experiments, exogenous human vWF (100 ug/mL, Hematologic Technologies) was added to washed platelets. Samples were washed three times in 1\% BSA in PBS and then stained with anti-rabbit 488 (1:250, Jackson Immunoresearch, West Grove, PA, USA) for $30 \mathrm{~min}$. After three washes in 1\% BSA in PBS, samples were resuspended in $300 \mu \mathrm{L}$ PBS and analyzed on a LSR Fortessa (BD Bioscience).

\section{Cryo-electron tomography}

Resting and sheared platelets in Tyrode buffer were seeded on gold grids coated with a silicon mesh (R 1/4, 200 mesh, Quantifoil, Jena, Germany). Platelets were allowed to adhere for $10 \mathrm{~min}$ and then fixed with $4 \%$ formaldehyde for $5 \mathrm{~min}$ at room temperature, before being processed for cryo-ET. GpIb and integrin $\alpha \operatorname{IIb} \beta 3$ receptors were detected by immunogold labeling (details are given in the Online Supplementary Data). Data were acquired using an FEI Titan Krios. Tomograms were acquired with a magnification of $42,000 \times$ corresponding to a pixel size of $0.34 \mathrm{~nm}$. The receptor density was analyzed using MATLAB scripts and the receptor distributions were plotted and statistically analyzed by OriginLab software (Northampton, MA, USA).

\section{Statistical analysis}

Data are plotted as the mean \pm standard error of mean of at least three independent experiments. They were analyzed by a paired, two-tailed Student $t$-test with GraphPad Prism version 7 (GraphPad Software, La Jolla, CA, USA). $P$ values $<0.05$ were considered statistically significant.

\section{Results}

Pre-incubation of blood with the n-3 FA ALA at dietary relevant concentrations ${ }^{23}$ reduced the $\mathrm{GpIb} / \mathrm{vWF}$ interaction under pathological high-shear flow $\left(10,000 \mathrm{~s}^{-1}\right.$, corresponding to the flow rate in an $80 \%$ stenosed artery), as measured by the platelet-covered area $(106,963 \pm 15,892$ $\mu \mathrm{m}^{2}$ with vehicle $v$ s. $75,519 \pm 16,254 \mu \mathrm{m}^{2}$ with ALA) (Figure 1A, B and Online Supplementary Videos S1 and S2). Analysis of single fluorescently labeled platelets showed that their speed was doubled when pre-incubated with ALA (Figure 1C), while the distance traveled before stopping was increased $(8.89 \pm 4.0 \mu \mathrm{m}$ with vehicle vs. $13.36 \pm 7.2 \mu \mathrm{m}$ with ALA) (Figure 1D, E).

It has been reported that GpIb resides in cholesterol-rich membrane domains termed lipid rafts, ${ }^{20,21,2,2,25}$ and that it appears to cluster in conditions of high-shear flow..$^{22}$ In agreement with these findings, cholesterol depletion with methyl- $\beta$-cyclodextrin $(M \beta C D)$, able to remove $50-90 \%$ of membrane cholesterol, ${ }^{26}$ greatly reduced platelet adhesion to vWF, demonstrating the pivotal role of membrane cholesterol in GpIb-vWF adhesion under high-shear flow $\left(188 \pm 16 \mu \mathrm{m}^{2}\right)$ (Figure 1F).

To exclude that the reduced adhesion of ALA-treated platelets was due to lower levels of membrane GpIb, vehicle- and ALA-treated washed platelets were exposed to high-shear flow in a viscometer (10,000 $\mathrm{s}^{-1}$ for $\left.1 \mathrm{~min}\right)$ and analyzed by flow cytometry. Levels of membrane Gplb were not different between vehicle- and ALA-treated platelets (Figure 2A); rather, pre-treatment with the n-3 FA preserved GpIb levels, as shown by higher fluorescence values, suggesting it had an inhibitory effect on GpIb cleavage, as previously shown by our group. ${ }^{19}$

Next, we analyzed whether the effect on GpIb-vWF binding was specific for $\mathrm{GpIb}$, vWF, or both. Whole blood was pre-treated with ALA or vehicle for $1 \mathrm{~h}$, followed by isolation of platelet-rich plasma and exposure to highshear flow (10,000 s-1 for $1 \mathrm{~min})$. Flow cytometric analysis of platelet-bound vWF showed that pathological highshear flow was able to induce GpIb-vWF binding, and that this was not influenced by the presence of ALA (Figure 2B). When the same experiment was performed with washed platelets, we could not detect any platelet-bound $\mathrm{vWF}$, demonstrating the plasmatic origin of the bound vWF (Figure 2C); however, addition of exogenous human $\mathrm{vWF}$ to washed platelets was able to restore vWF-platelet binding (Figure 2C). These results show that ALA has no effect on vWF itself, and suggest that its inhibitory effect is exerted through platelet GpIb and is specific for binding to anchored $\mathrm{vWF}$ (as typically exposed in vivo by endothelial cells after injury).

High resolution imaging of $\mathrm{GpIb}$ receptors at the plasma membrane of intact platelets was conducted using cryo$\mathrm{ET}^{27}$ (Figure 3A-C). Adherent platelets were incubated with anti-GpIb antibodies decorated with 6-nm gold-protein $\mathrm{A}$ and imaged with cryo-ET. The coordinates of the gold nanoclusters were identified (Figure 3A-C). While the 
S. Stivala et al.

A

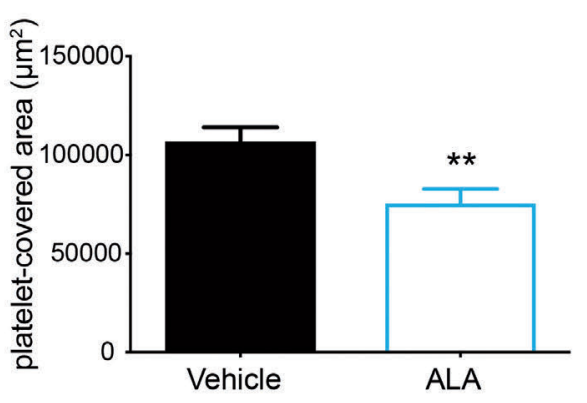

C

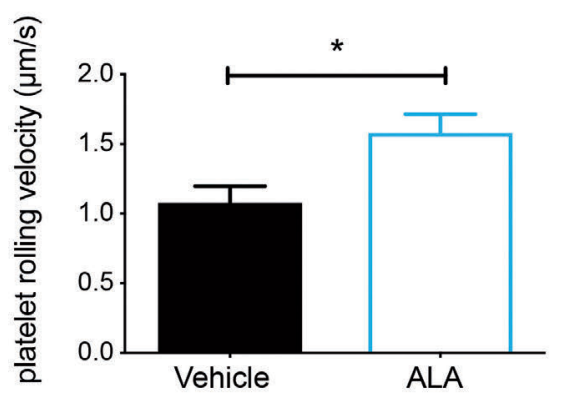

E

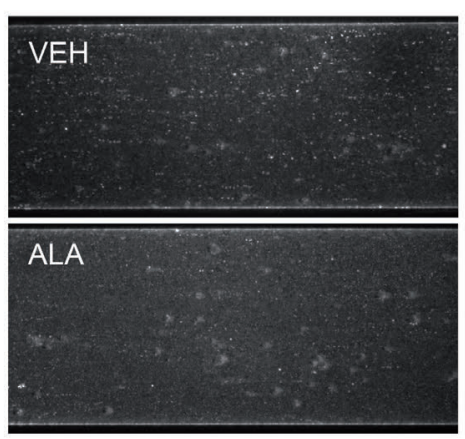

B

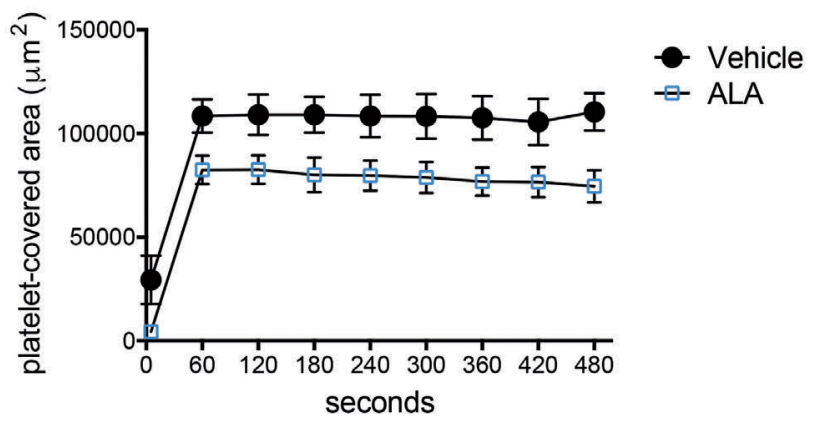

D

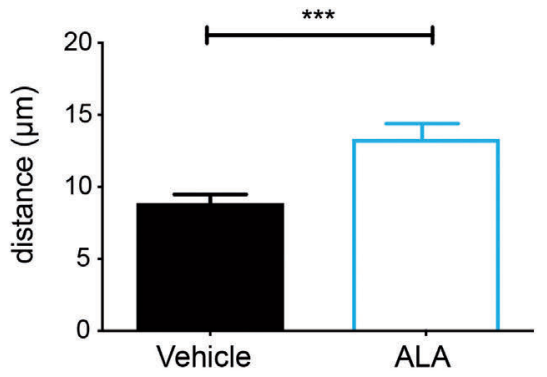

$\mathbf{F}$

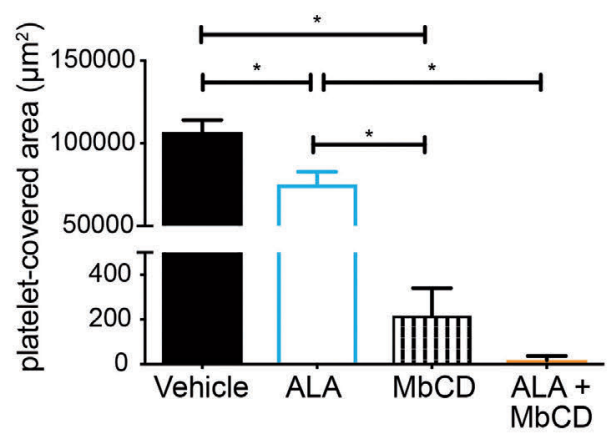

Figure 1. Platelet adhesion to won Willebrand factor is inhibited by $\alpha$-linolenic acid. Blood collected into EDTA and pre-incubated with vehicle or $\alpha$-linolenic acid (ALA) for $1 \mathrm{~h}$ was perfused on won Willebrand factor (VWF) at high-shear rate and platelet adhesion monitored by fluorescence microscopy. Platelet-covered area measured at the end of the perfusion (A) or every minute during the perfusion (B) was significantly reduced by the ALA treatment ( $n=6, P=0.0039$ ). The first time-point in $B$ corresponds to $5 \mathrm{~s}$ after the start of the flow. (C) Single platelet rolling velocity measurements showed an increased speed in the ALA-treated samples, and a carespondingly increased distance until firm adhesion (D) ( $n=6, P=0.04$ for $C$ and $P=0.0009$ for $D$ ). (E) Representative projection images of a vehicle (VEH)- or ALA-treated sample showing single platelets rolling over vWF. (F) Membrane cholesterol depletion by pre-incubation of EDTA-blood with methyl- $\beta$ cyclodextrin MbCD) completely abolished platelet adhesion to VWF $(n=6, P<0.0001)$.

overall number of receptors per platelet did not differ significantly $\left(115 \pm 50\right.$ per $\mu \mathrm{m}^{2}$ for the adherent platelets $v s$. $130 \pm 50$ per $\mu \mathrm{m}^{2}$ in the case of the shear-activated platelets), the distribution of receptors varied. To quantify these changes, we analyzed the clustering properties of GlIb $\alpha$ by calculating, for each receptor, the number of receptors within a $50 \mathrm{~nm}$ radius. Figure $3 \mathrm{E}-\mathrm{G}$ shows normail distributions of neighborhood receptors, with an average of $2.5 \pm 1.8$ neighbors for the adherent platelets, $3 \pm 2.3$ neighbors for the shear-activated platelets and $3 \pm 1.8$ for ALA-treated platelets. While the global distribution of receptors remained similar, $9 \%$ of the receptors (41/453) in the shear-activated platelets had $\geq 12$ neighbors (Figure $3 \mathrm{~F})$, while in the case of platelets treated with ALA only $3.8 \%$ of receptors $(14 / 366)$ had more than 12 neighbors (Figure 3G). As a control, we analyzed the density and number of neighbors of the platelet integrin $\alpha \operatorname{Ilb} \beta 3$. This analysis showed a much higher density of integrin receptors $\left(450 \pm 180\right.$ per $\left.\mu \mathrm{m}^{2}\right)$ and with many neighbors in comprison to the GuIb receptors $\left(10 \pm 5\right.$ per $\left.\mu \mathrm{m}^{2}\right)$ (Figure $3 \mathrm{D}$, $\mathrm{H}$ and Online Supplementary Figure S3), which is in agreemont with the difference in abundance of the two receptors in platelets. ${ }^{28}$

Analysis of immunofluorescent-stained GuIb $\alpha$ by GSD super-resolution microscopy also revealed that $\mathrm{GpIb}$ is abundantly expressed over the whole platelet membrane with small local points of high density (Figure 4A). To compare the GSD microscopy and cryo-ET findings, we adopted a recently developed strategy. ${ }^{29}$ We used an assembly of the cryo-ET data into a $5 \mu \mathrm{m}$ x $5 \mu \mathrm{m}$ collage in which the coordinates of the $6 \mathrm{~nm}$ gold-labeled GuIb were drawn as red dots and blurred to $20 \mathrm{~nm}$ resolution to

1662

haematological | 2020; 105(6) 
A

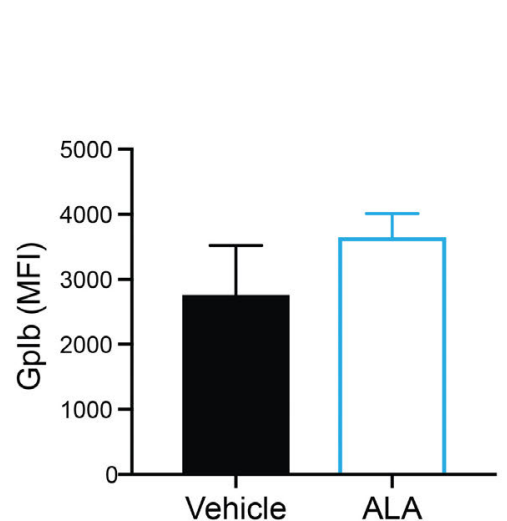

B

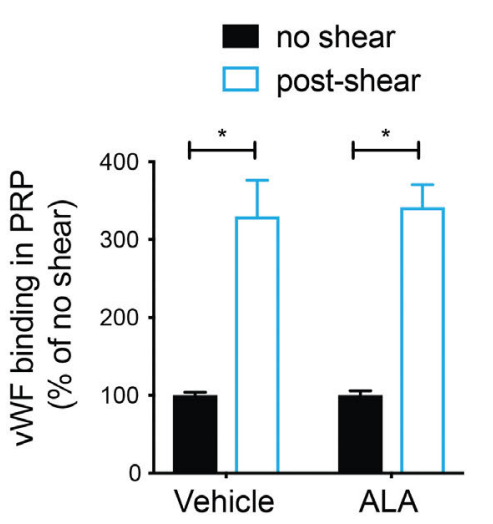

C

Vehicle

ALA

QD WP+VWF

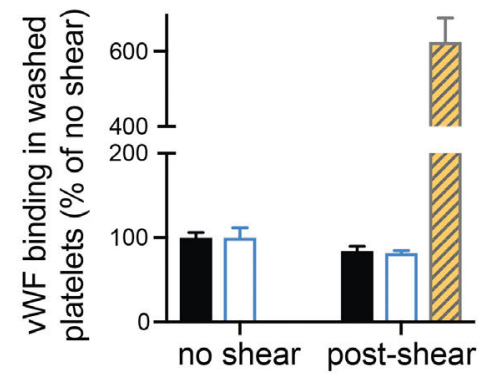

Figure 2. High-shear induces von Willebrand factor binding in platelet-rich plasma but not in washed platelets. (A) Washed platelets were exposed to high-shear flow and analyzed for Gplb abundance by flow cytometry, which did not reveal any difference between vehicle- and $\alpha$-linolenic acid (ALA)-treated platelets ( $n=3$ ). MFI: mean fluorescence intensity. (B) Platelet-rich plasma was either left untreated or exposed to high-shear flow, and platelet-bound von Willebrand factor (vWF) analyzed by flow cytometry. In both vehicle- and ALA-treated samples, high-shear flow induced vWF binding, as shown by a 3-fold increase compared to that of samples not exposed to the shear force $(n=3, P<0.05)$. (C) Exposing washed platelets (WP) to high-shear flow did not lead to vWF binding; addition of exogenous human vWF before shear exposure resulted in robust binding $(n=3)$.

match the GSD data (Figure 4B). The obtained synthetic model of platelet perimeter showed a distribution and appearance of the receptor in agreement with the fluorescence data, with a high density of GpIb throughout the membrane, but, interestingly, also local, small regions of more densely packed complexes (Figure 4B, arrowhead). Pre-treating blood with ALA did not cause a change in the distribution of membrane GpIb detected by total internal reflection fluorescence (TIRF), likely due to the small size of GpIb clusters, which is in the same range as the resolution of the technique (Figure 5).

\section{Discussion}

ALA is a plant-derived n-3 FA readily available in certain plant oils such as flaxseed, soybean and canola oil. Epidemiological studies have shown an inverse correlation between dietary ALA and cardiovascular events, ${ }^{11,30,31}$ although the molecular mechanisms of this protection are not completely known. Our group has investigated the molecular basis of several cardio-protective effects of ALA, showing that at least some of its effects are mediated by its action on endothelial cells, leukocytes and platelets.,10,19 In this study, we have focused in particular on platelet adhesion to vWF under high-shear conditions, which represents the first step mediating platelet activation under arterial flow and is especially important in stenosed (atherosclerotic) arteries, where shear can reach extremely high values $\left.\left(>5,000 \mathrm{~s}^{-1}\right) \cdot\right)^{7,32,33}$

Here we show for the first time that ALA is able to partially inhibit platelet adhesion to vWF under a shear flow of $10,000 \mathrm{~s}^{-1}$, when whole blood is pre-incubated for $1 \mathrm{~h}$ with ALA at dietary relevant concentrations. ${ }^{23}$ This is accompanied by an increase in speed and distance traveled by ALA-treated platelets, compared to vehicle-treated platelets (Figure 1), and extends previous results from our group showing a reduced aggregation of citrated platelets over collagen at low shear. ${ }^{19}$ The effect observed is specific to anchored vWF, since vWF binding upon exposure of platelet-rich plasm to high-shear flow could not be altered by ALA pre-treatment (Figure 2). A similar inhibition of platelet adhesion was obtained with the longer, marinederived n-3 FA eicosapentaenoic acid, while the saturated fatty acid stearic acid had no effect (Online Supplementary Figure S4). Although the inhibitory effect of ALA may seem small (25\% reduction in platelet adhesion), its biological implications are important, since inhibition at this early step will reduce the number of platelets activated in response to $\mathrm{GpIb}$ engagement and subsequent signaling leading to thrombus formation. The additional inhibitory effects of ALA at the level of intracellular signaling and granule secretion will lead, overall, to a greater effect with a relevant biological significance on atherothrombosis.

Since platelet binding to vWF is mediated by the GpIb receptor, we employed state-of-the-art methods (superresolution microscopy by GSD and cryo-ET) to analyze GpIb distribution on the platelet membrane at high-resolution.

The GpIb distribution analysis by cryo-EI suggests that a significant subpopulation of receptors clustered into a high number of neighbours in the shear activated (and to a lesser extent in the ALA-treated) platelets, indicating that platelet exposure to shear stress induces a local rearrangement of $\mathrm{GpIb}$ at the platelet plasma membrane, forming discrete small regions of high receptor density (Figure 3). These regions presumably represent high affinity "binding units" or even binding loci for anchoring multimeric vWF.

Our observations are in line with previous findings ${ }^{34}$ showing that under high-shear stress platelets form local points of adhesion, termed "discrete adhesion points", which are the putative regions of interaction between platelets/vWF. Our functional and structural data provide a high-resolution insight into the position of the GpIb receptors and support a model where high-shear stress induces a rapid, local rearrangement of GpIb receptors into small "clusters" of 15-20 complexes. This subpopulation of receptors may represent the previously described high-affinity binding units that interact with vWF and enable platelet rolling under arterial flow. Pre-incubation of platelets with ALA reduces the local clustering of $\mathrm{GpIb}$ receptors as shown by the virtual absence of larger complexes (GPIb with $\geq 17$ neighbors) (Figure $3 \mathrm{C}, \mathrm{G}$ ). On the 

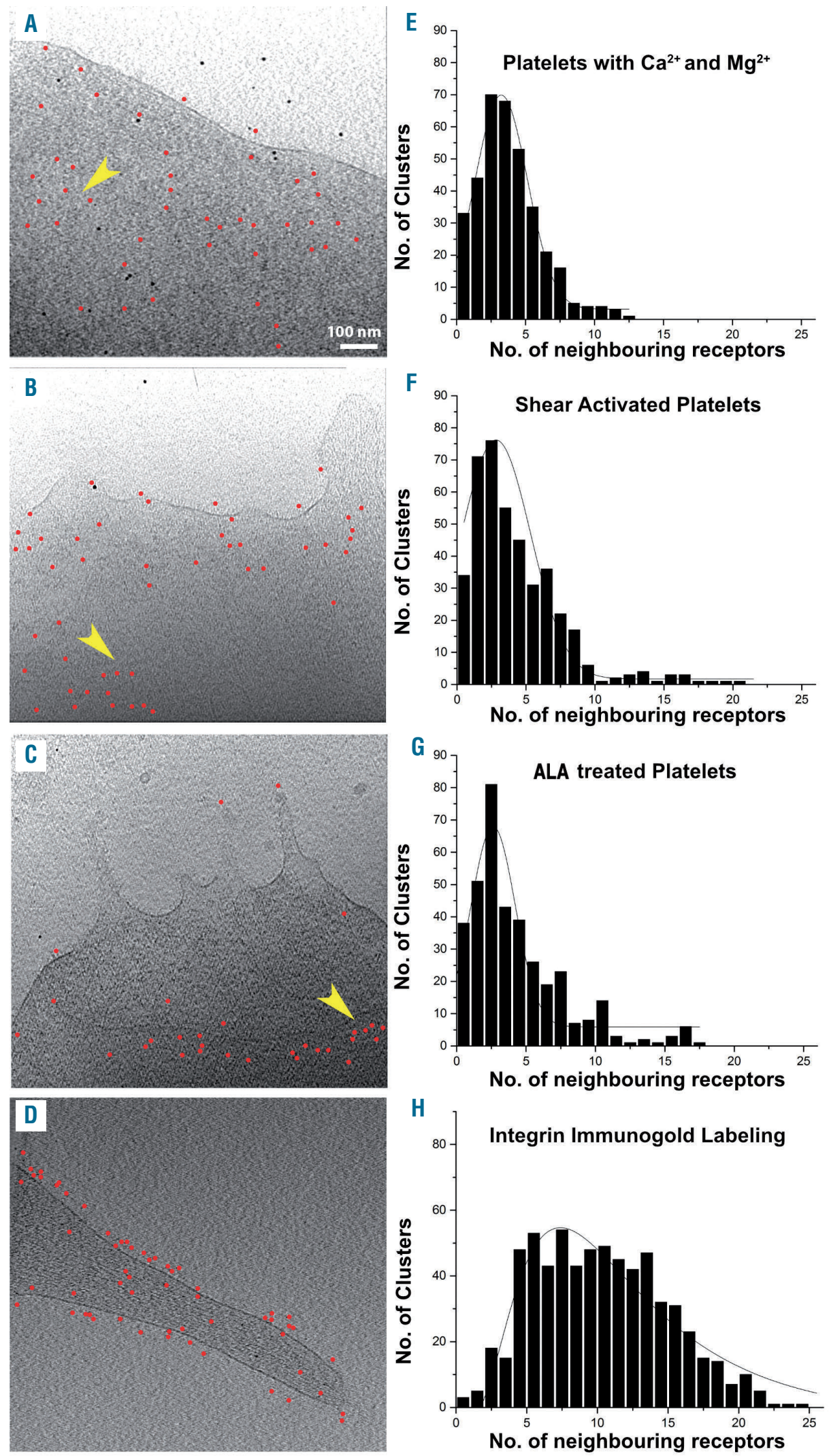

No. of neighbouring receptors

Figure 3. High-shear induces a local rearrangement of Gplb as revealed by cryo-electron tomography. (A-C) Immunogold labeling and (E-G) neighborhood analysis for the control (A, E), shear-activated (B, F) and $\alpha$-linolenic acid (ALA)-treated shear-activated (C, G) platelets. (A-C): Projection images ( 70 nm in thickness) obtained from tomograms of platelets on which Gplba has been labeled with immunogold. The labeling was detected with protein $\mathrm{G}$ conjugated to 6 nm gold. To make the gold identification easier, the gold has been labeled with red circles. The yellow arrows indicate crowded neighborhoods in the pictures. All the projections have the same scale and the scale bar in A is $100 \mathrm{~nm}$. (E-G) Analysis of the immunogold labeling. The three-dimensional coordinates of the $6 \mathrm{~nm}$ gold particles from six tomograms for each condition were selected and the distances to the "neighboring receptors" within a radius of $50 \mathrm{~nm}$ were calculated. The number of neighbors each receptor has is depicted in the histogram. (D) Integrin $\alpha$ llb $\beta 3$ immunogold labeling was performed on spread platelets and $(\mathrm{H})$ the same neighboring analysis performed. 
functional side, this provides an explanation as to why adhesion is significantly inhibited by ALA pre-treatment while platelet rolling speed is increased (Figure 1A, C). Previous work has shown that $\omega-3$ FA (of marine origin) can inhibit protein palmitoylation and, therefore, localization to lipid rafts..$^{35}$ Although in our experiments the preincubation time was too short to achieve an analog effect, long-term, nutritional supplementation with ALA may also inhibit GpIb localization to lipid rafts via reduced palmitoylation and, consequently, reduce platelet adhesion to vWF even more through this additional mechanism.

Taken together, these data provide insight into the possible mechanism of the anti-thrombotic properties of $n-3$ FA in the early phase of thrombosis at sites of arterial stenosis or plaque. It may therefore represent the basis for a therapeutic approach that interferes with this process.

In conclusion, our structural data from intact platelets,
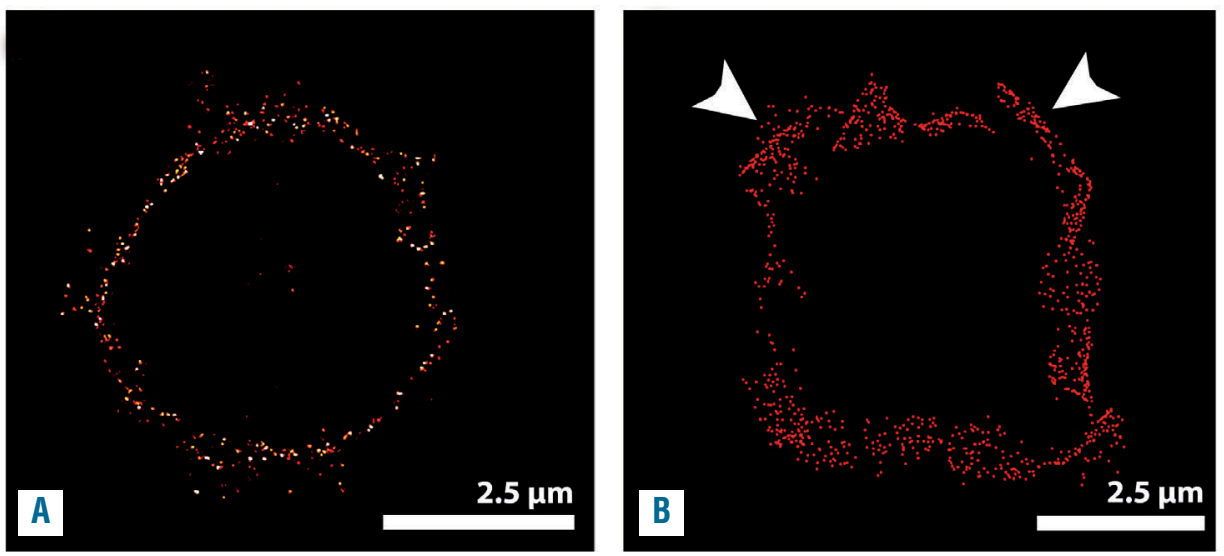

Figure 4. Comparison of Gplb labeling by super-resolution microscopy and cryo-electron tomography reveals a similar receptor distribution. (A) The Gplb receptors in shear-activated platelets were labeled and imaged by ground state depletion (GSD) as described in the Methods. (B) In order to compare the localization of the receptors determined by cryo-electron tomography and GSD microscopy, a synthetic model of platelet perimeter was generated by merging 17 tomograms from the gold-labeled platelets. The gold particle coordinates from the synthetic platelets were filtered to the same resolution as the GSD $(20 \mathrm{~nm})$, represented here in red. White arrowheads indicate local clustering areas. Scale bar: $2.5 \mu \mathrm{m}$ for both $A$ and $B$.
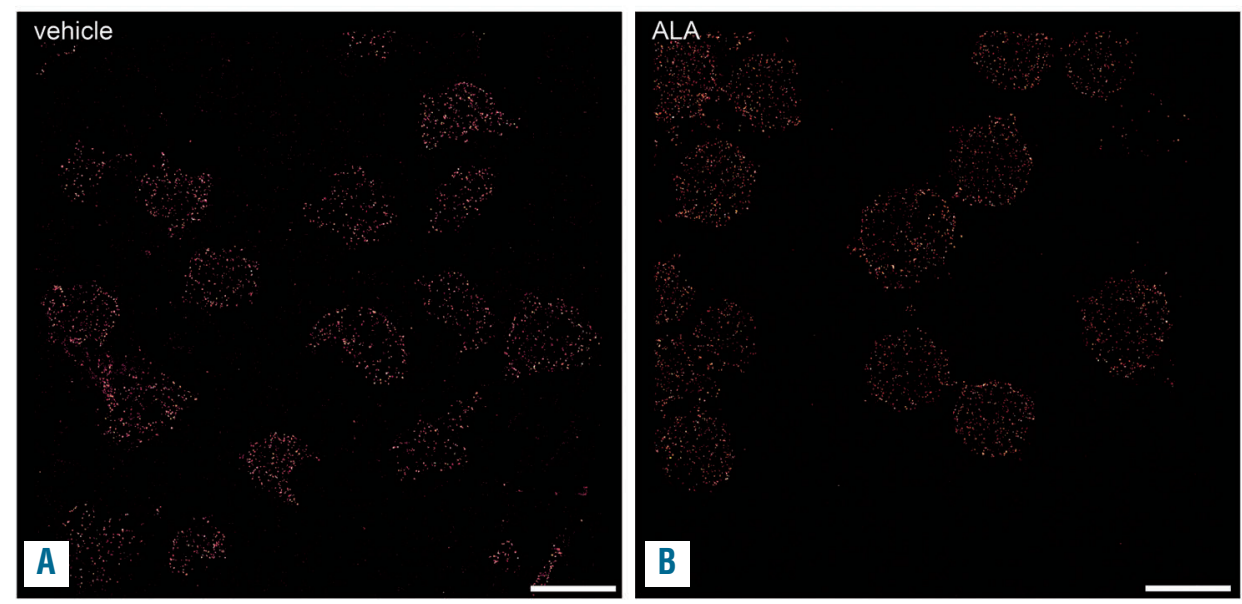

Figure 5. Analysis of Gplb distribution by total internal reflection microscopy. (A-D) Superresolution analysis of Gplb in platelets treated with vehicle (A), $\alpha$ linolenic acid (ALA) (B) or methyl- $\beta$ cyclodextrin (MbCD) (C) showed no detectable differences in the distribution of the receptor as measured by particle size (D). Scale bar: $2.5 \mu \mathrm{m}$.
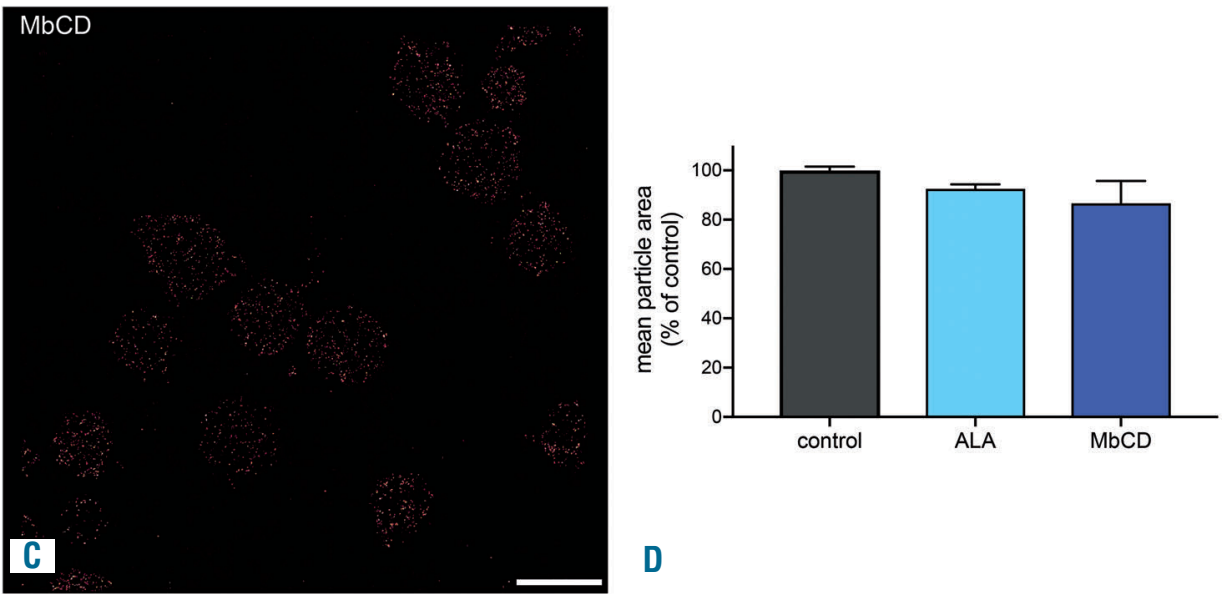
including cryo-ET and super-resolution microscopy, show that upon high-shear conditions platelet GpIb receptors reorganize into "clusters" of 10 to 20 complexes closer to each other than on resting platelets; functionally, we demonstrate that, through intervention with the plantderived n-3 FA ALA, the size of these clusters is decreased, thereby reducing platelet adhesion to vWF under highshear flow. This highlights the potential of ALA as an antithrombotic agent.

\section{Acknowledgments}

This work was supported by a grant from the Swiss National Foundation of Science, the Swiss Heart Foundation, the Kardio Foundation, Switzerland, and the Budai Foundation, Lichtenstein, to JHB (grant n. 310030_144152), and the Mäxi Foundation to OM. We are grateful to Prof. B. Coller, New York, for the generous gift of the 6D1 antibody against human GpIb. We also thank the Center for Microscopy and Image Analysis at the University of Zurich (ZMB). Switzerland.

\section{References}

1. Canobbio I, Balduini C, Torti M. Signalling through the platelet glycoprotein Ib-V-IX complex. Cell Signal. 2004;16(12):13291344.

2. Bergmeier W, Piffath CL, Goerge T, et al. The role of platelet adhesion receptor GPIbalpha far exceeds that of its main ligand, von Willebrand factor, in arterial thrombosis. Proc Natl Acad Sci U S A. 2006;103(45):16900-16905

3. Englund GD, Bodnar RJ, Li Z, Ruggeri ZM, Du X. Regulation of von Willebrand factor binding to the platelet glycoprotein Ib-IX by a membrane skeleton-dependent insideout signal. J Biol Chem. 2001;276(20): 16952-16959.

4. Yago T, Lou J, Wu T, et al. Platelet glycoprotein Ib forms catch bonds with human WT vWF but not with type 2B von Willebrand disease vWF. J Clin Invest. 2008;118(9): 3195-3207.

5. Fu H, Jiang Y, Yang D, Scheiflinger F, Wong WP, Springer TA. Flow-induced elongation of von Willebrand factor precedes tensiondependent activation. Nat Commun. 2017;8(1):324

6. Butera D, Passam F, Ju L, et al. Autoregulation of von Willebrand factor function by a disulfide bond switch. Sci Adv. 2018;4(2):eaaq1477.

7. Sakariassen KS, Orning L, Turitto VT. The impact of blood shear rate on arterial thrombus formation. Future Sci OA. 2015;1(4):FSO30.

8. Casa LDC, Deaton DH, Ku DN. Role of high shear rate in thrombosis. J Vasc Surg. 2015;61(4):1068-1080.

9. Winnik S, Lohmann C, Richter EK, et al. Dietary $\alpha$-linolenic acid diminishes experimental atherogenesis and restricts $\mathrm{T}$ celldriven inflammation. Eur Heart J. 2011;32 (20):2573-2584.

10. Holy EW, Forestier M, Richter EK, et al. Dietary $\alpha$-linolenic acid inhibits arterial thrombus formation, tissue factor expression, and platelet activation. Arterioscler Thromb Vasc Biol. 2011;31(8):1772-1780.

11. Campos H, Baylin A, Willett WC. Alphalinolenic acid and risk of nonfatal acute myocardial infarction. Circulation. 2008; 118(4):339-345.

12. Sala-Vila A, Guasch-Ferré $\mathrm{M}, \mathrm{Hu} \mathrm{FB}$, et al. Dietary $\alpha$-linolenic acid, marine $\omega-3$ fatty acids, and mortality in a population with high fish consumption: findings from the PREvención con DIeta MEDiterránea
(PREDIMED) study. J Am Heart Assoc. 2016;5(1):1-12

13. Guallar E, Sanz-Gallardo MI, van't Veer P, et al. Mercury, fish oils, and the risk of myocardial infarction. N Engl J Med. 2002;347(22):1747-1754.

14. Mozaffarian D, Rimm EB. Fish intake, contaminants, and human health: evaluating the risks and the benefits. JAMA. 2006;296(15):1885-1899.

15. Morris MC, Brockman J, Schneider JA, et al. Association of seafood consumption, brain mercury level, and APO\&4 status with brain neuropathology in older adults. JAMA. 2016;60612(5):489-497.

16. Shaikh SR, Jolly CA, Chapkin RS. n-3 Polyunsaturated fatty acids exert immunomodulatory effects on lymphocytes by targeting plasma membrane molecular organization. Mol Aspects Med. 2012;33(1):46-54.

17. Shaikh SR, Rockett BD, Salameh M, Carraway K. Docosahexaenoic acid modifies the clustering and size of lipid rafts and the lateral organization and surface expression of MHC class I of EL4 cells. J Nutr. 2009;139(9):1632-1639.

18. Chen W, Jump DB, Esselman WJ, Busik J V. Inhibition of cytokine signaling in human retinal endothelial cells through modification of caveolae/lipid rafts by docosahexaenoic acid. Invest Ophthalmol Vis Sci 2007;48(1):18-26

19. Stivala S, Reiner MF, Lohmann C, Lüscher TF, Matter CM, Beer JH. Dietary $\alpha$-linolenic acid increases the platelet count in ApoE-/mice by reducing clearance. Blood. 2013;122(6):1026-1033

20. Shrimpton CN, Borthakur G, Larrucea S, Cruz MA, Dong J-F, Lopez JA. Localization of the adhesion receptor glycoprotein IbIX-V complex to lipid rafts is required for platelet adhesion and activation. J Exp Med. 2002;196(8):1057-1066.

21. Munday AD, Gaus K, López JA. The platelet glycoprotein Ib-IX-V complex anchors lipid rafts to the membrane skeleton: implications for activation-dependent cytoskeletal translocation of signaling molecules. J Thromb Haemost. 2010;8(1):163172 .

22. Gitz E, Koopman CD, Giannas A, et al. Platelet interaction with von Willebrand factor is enhanced by shear-induced clustering of glycoprotein Ib $\alpha$ Haematologica. 2013;98(11):1810-1818.

23. Harper CR, Edwards MJ, DeFilipis AP, Jacobson TA. Flaxseed oil increases the plasma concentrations of cardioprotective (n-3) fatty acids in humans. J Nutr. 2006;136(1):83-87.

24. Canobbio I, Trionfini P, Guidetti GF Balduini C, Torti M. Targeting of the small GTPase Rap2b, but not Rap1b, to lipid rafts is promoted by palmitoylation at Cys 176 and Cys177 and is required for efficient protein activation in human platelets. Cell Signal. 2008;20(9):1662-1670.

25. Bodin $\mathrm{S}$, Tronchère $\mathrm{H}$, Payrastre B. Lipid rafts are critical membrane domains in blood platelet activation processes Biochim Biophys Acta. 2003;1610(2):247257.

26. Gousset K, Wolkers WF, Tsvetkova NM, et al. Evidence for a physiological role for membrane rafts in human platelets. J Cell Physiol. 2002;190(1):117-128.

27. Sorrentino S, Studt JD, Horev MB, Medalia O, Sapra KT. Toward correlating structure and mechanics of platelets. Cell Adhes Migr. 2016;10(5):568-575.

28. Lewandrowski U, Wortelkamp S, Lohrig K, et al. Platelet membrane proteomics: a novel repository for functional research. Blood. 2009;114(1):e10-e19.

29. Turgay Y, Eibauer M, Goldman AE, et al The molecular architecture of lamins in somatic cells. Nature. 2017;543(7644):261264.

30. Albert CM, Oh K, Whang W, et al. Dietary alpha-linolenic acid intake and risk of sudden cardiac death and coronary heart disease. Circulation. 2005;112(21):3232-3238.

31. Djoussé L, Arnett DK, Carr JJ, et al. Dietary linolenic acid is inversely associated with calcified atherosclerotic plaque in the coronary arteries: the National Heart, Lung, and Blood Institute Family Heart Study. Circulation. 2005;111(22):2921-2926.

32. Bark DL, Ku DN. Wall shear over high degree stenoses pertinent to atherothrombosis. J Biomech. 2010;43(15):2970-2977.

33. Strony J, Beaudoin A, Brands D, Adelman B. Analysis of shear stress and hemodynamic factors in a model of coronary artery stenosis and thrombosis. Am J Physiol. 1993;265(5 Pt 2):H1787-1796.

34. Reininger AJ, Heijnen HFG, Schumann H, Specht HM, Schramm W, Ruggeri ZM. Mechanism of platelet adhesion to von Willebrand factor and microparticle formation under high shear stress. Blood. 2006;107(9):3537-3545.

35. Webb Y, Hermida-Matsumoto L, Resh MD Inhibition of protein palmitoylation, raft localization, and $\mathrm{T}$ cell signaling by 2-bromopalmitate and polyunsaturated fatty acids. J Biol Chem. 2000;275(1):261-270. 